\title{
Electrospray/VUV Single-Photon Ionization \\ Mass Spectrometry for the Analysis of Organic Compounds
}

\author{
Zheng Yang, ${ }^{\text {a }}$ Taichang Zhang, ${ }^{\text {b }}$ Yang Pan, ${ }^{\text {b }}$ Xin Hong, ${ }^{\text {b }}$ Zichao Tang, ${ }^{\text {a,c }}$ \\ and Fei Qi $\mathrm{i}^{\mathrm{b}}$ \\ a State Key Laboratory of Molecular Reaction Dynamics, National Laboratory of Molecular Sciences, Institute of \\ Chemistry, Chinese Academy of Sciences, Beijing, P.R. China \\ ${ }^{\mathrm{b}}$ National Synchrotron Radiation Laboratory, University of Science and Technology of China, Hefei, P.R. China \\ ' State Key Laboratory of Molecular Reaction Dynamics, Dalian Institute of Chemical Physics, Chinese Academy of \\ Sciences, Dalian, P.R. China
}

For the comprehensive analysis of organic compounds, especially thermal labile and nonpolar compounds, an electrospray/vacuum ultraviolet (VUV) single-photon ionization (ES-SPI) method was developed. The fine droplets of the sample solution from the electrospray process were directed through a quartz capillary and two skimmers to form a molecular beam into a high vacuum ionization chamber. The neutral sample molecules were softly ionized with tunable VUV light and analyzed with a reflection time-of-flight mass spectrometer (RTOF-MS). The ionization energy (IE) and appearance onsets of fragments were obtained based on the photoionization efficiency (PIE) spectrum. The isomers can also be distinguished. With this new method, clean (fragment-free) mass spectra of nonpolar compounds, such as benzene, cyclohexane, and some thermal labile solid compounds (triphenylamine, thioacetamide, and urea) have been obtained without any tedious pretreatment. The components of complex mixtures (gasoline and kerosene) can be identified. Furthermore, quantitative analysis of the components can be obtained based on photoionization cross section data. This method may be used for quantitative analysis of small biomolecules and natural products. (J Am Soc Mass Spectrom 2009, 20, 430-434) (c) 2009 Published by Elsevier Inc. on behalf of American Society for Mass Spectrometry

$\mathrm{E}$ lectrospray is an important method to bring intact molecules from solution into the gas phase. However, electrospray ionization mass spectrometry (ESI-MS) has difficulty in detecting weak polar and nonpolar compounds, such as hydrocarbons in petroleum [1-4]. Photoionization (PI) is an alternative ionization process relatively nondiscriminatory to nonpolar compounds. VUV single-photon ionization (SPI) is especially useful for fragment-free ionization of organic compounds. Tunable synchrotron VUV SPI time-offlight mass spectrometer (TOF-MS) has been used for analysis of organic samples in the gas phase [5-7]. VUV SPI mass spectrometry [8-10] with tunable lamps or lasers enables distinction of components of complex mixtures, including isomers [11]. The combination of electrospray and VUV SPI can be a very powerful tool for investigating organic compounds or biological molecules. Here, we report the first electrospray-VUV singlephoton ionization (ES-SPI) studies of low polarity and thermal labile compounds.

Address reprint requests to Zichao Tang, Dalian Institute of Chemical Physics, Zhongshan Road no. 457, Dalian 116023, PR China. E-mail: zctang@dicp.ac.cn

\section{Experimental}

The experiments were performed in the National Synchrotron Radiation Laboratory (NSRL), Hefei, China. The interface to the synchrotron has been reported elsewhere [11]. A detailed diagram of our ion source is shown in Figure 1. The source consists of a syringe pump (KD Scientific 101, Holliston, MA, USA), a $0.1 \mathrm{~mm}$ i.d. stainless steel spray needle (the ES voltage applied normally from 1000 to $3000 \mathrm{~V}$ ), a $150 \mathrm{~mm}$ long quartz capillary $(1.0 \mathrm{~mm}$ i.d., $10 \mathrm{~mm}$ o.d.) with a grounded stainless steel sleeve at its inlet (serving as the counterelectrode), and a sample chamber with a quartz skimmer of $0.5 \mathrm{~mm}$ orifice. The distance between the quartz capillary and the quartz skimmer was $6 \mathrm{~mm}$.

The flow rates of the sample solution ranged from 0.1 to $100 \mu \mathrm{L} / \mathrm{min}$, controlled by the syringe pump. In this work, the quartz capillary was heated to $90^{\circ} \mathrm{C}$ to vaporize the sample droplets. A $5 \mathrm{D}$ platform and a microscope were used to adjust the position of the spray needle relative to the quartz capillary. Chamber I between the exit of the quartz capillary and the quartz skimmer was pumped with a $70 \mathrm{~L} / \mathrm{s}$ roots pump backed with a $27 \mathrm{~L} / \mathrm{s}$ mechanical pump. The typical working vacuum of Chamber I was $120 \mathrm{~Pa}$.
(C) 2009 Published by Elsevier Inc. on behalf of American Society for Mass Spectrometry. $1044-0305 / 09 / \$ 32.00$

doi:10.1016/j.jasms.2008.10.026
Published online November 21, 2008 Received July 9, 2008 Revised October 15, 2008 Accepted October 31, 2008 


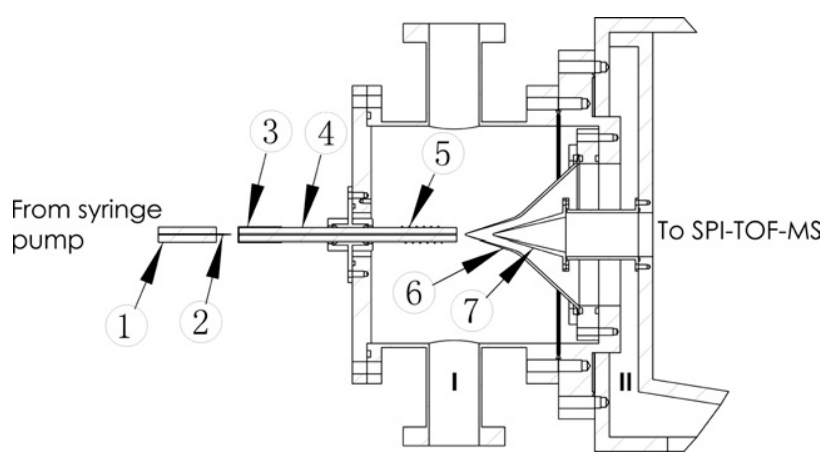

Figure 1. Schematic of the electrospray sample inlet interface: (1) insulating holder; (2) ES needle; (3) stainless steel sleeve as ES counterelectrode (grounded); (4) quartz capillary; (5) heater wire; (6) quartz skimmer; (7) nickel skimmer.

Here, electrospray served as a method for transmission of the samples into the gas phase, but not primarily for ionization. Therefore, the ES parameters such as the voltage of the spray needle, the flow rate of the sample solution, and the distance between the needle and the counterelectrode could vary relatively widely and still provide good ion signal. In this work, relatively small distance, low ES voltage, and high flow rate (compared with conventional ESI) were used to obtain stable electrospray, good sampling efficiency, and strong SPI signals.

Chamber II was pumped with a $3500 \mathrm{~L} / \mathrm{s}$ turbo molecular pump backed with a $70 \mathrm{~L} / \mathrm{s}$ roots pump and a $15 \mathrm{~L} / \mathrm{s}$ mechanical pump. The working pressure of Chamber II was about $5 \times 10^{-3} \mathrm{~Pa}$. The molecular beam passed through the quartz skimmer was sampled by a nickel skimmer ( $2 \mathrm{~mm}$ orifice, $18 \mathrm{~mm}$ downstream from the quartz skimmer) at the end of this chamber and went into the photoionization chamber (working pressure typically $2 \times 10^{-3} \mathrm{~Pa}$ ).

A detailed description of the analyzer was published elsewhere [11]. Briefly, a $1 \mathrm{~m}$ home-built Seya-Namioka monochromator with a grating of 1200 grooves $/ \mathrm{mm}$ was used to disperse the synchrotron radiation from the bending beam line of the $800 \mathrm{MeV}$ electron storage ring. The energy resolving power $\mathrm{E} / \Delta \mathrm{E}(\mathrm{FWHM})$ of VUV was about 500 with the photon flux of about $5 \times 10^{10}$ photons/s. A lithium fluoride ( $\mathrm{LiF}$ ) window of $1.0 \mathrm{~mm}$ thickness was used to eliminate the higher-order radiation emerging from the monochromator. The photon flux was monitored by a silicon photodiode (SXUV-100; International Radiation Detectors Inc., Torrance, CA, USA) to normalize the ion signal. After ionization, a pulsed voltage of $385 \mathrm{~V}$ was used to drive the ions into the flight tube which was floated at $-2000 \mathrm{~V}$. The ions were reflected by the reflection plates and were detected with a micro-channel plate (MCP) detector. The ion signals were amplified by a pre-amplifier (VT120C; Ortec, Oak Ridge, TN, USA), and the TOF mass spectra were recorded with a P7888 multichannel scaler (FAST Comtec, Germany) using a 2 ns bin width. A digital delay generator (DG535, Stanford Research Systems,
Inc., Sunnyvale, CA, USA) was used to trigger the repelling voltage pulse and the multichannel scaler. The repetition rate of the sampling was 10k Hz. The resolution of the RTOF-MS was $\sim 1400$. The photoionization efficiency (PIE) spectra were acquired by scanning the VUV wavelength.

Methanol $(\geq 99.7 \%)$, acetonitrile $(\geq 99.0 \%)$, benzene ( $\geq 99.5 \%)$, toluene $(\geq 99.5 \%)$, cyclohexane $(\geq 99.5 \%)$, nitrobenzene $(\geq 99.5 \%)$, thioacetamide $(\geq 99.0 \%)$, triphenylamine $(\geq 99.9 \%)$, and urea $(\geq 99.0 \%)$ were purchased from Sinopharm Chemical Reagent Co., Ltd, Shanghai, China. The no. 90 standard unblended gasoline [12] and the kerosene in this work were purchased from Fangyuan Inc., Liaoning, China; the kerosene was used as an unknown mixture sample. No further purification was performed. Solvents with ionization potential higher than those of the samples were used to ensure that the solvent molecules do not interfere with the mass signal of the samples.

\section{Results and Discussion}

\section{Low Polar/Nonpolar Liquid Compounds}

This ES-SPI method was tested at $10.50 \mathrm{eV}$ photon energy with a methanol solution containing a mixture of benzene, cyclohexane, toluene, and nitrobenzene. The concentrations of the solutes were $\sim 0.1 \mathrm{~mol} / \mathrm{L}$ each and the flow rate was $15 \mu \mathrm{L} / \mathrm{min}$. The voltage applied on the ES needle was $1500 \mathrm{~V}$. The distance between the needle and the counterelectrode was $1.0 \mathrm{~mm}$. The mass peaks at $m / z=78,84,92$, and 123 in Figure 2 correspond to benzene, cyclohexane, toluene, and nitrobenzene, respectively. Very little fragments appear in the mass spectrum.

The detection limit (DL) of toluene can be estimated with the following equation [13]:

$$
D L=2 \delta c / h
$$

where $c$ is the concentration of the toluene, $\delta$ is the standard deviation of the noise, and $h$ is the toluene

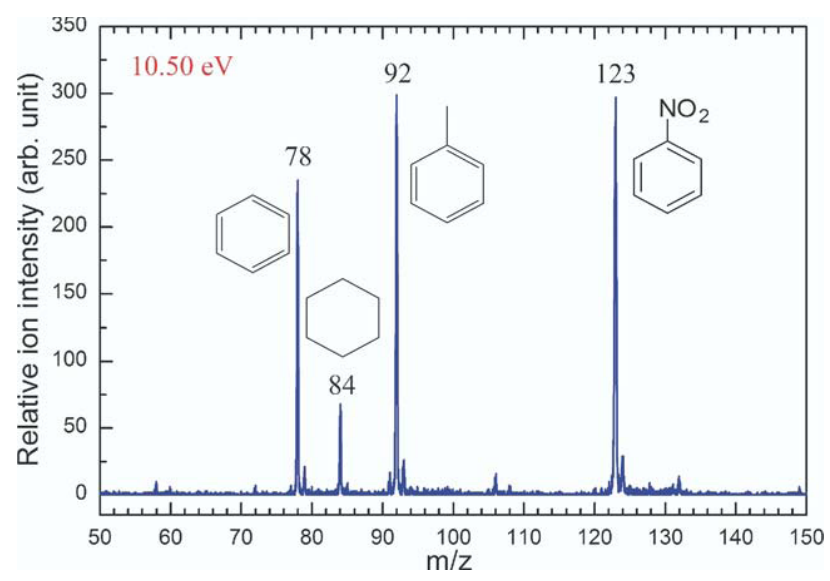

Figure 2. ES-SPI mass spectrum of a methanol solution containing benzene, cyclohexane, toluene, and nitrobenzene at $10.50 \mathrm{eV}$ photon energy. The concentrations of the solutes are $\sim 0.1 \mathrm{~mol} / \mathrm{L}$. 
peak height in the spectrum. In this experiment, the toluene peak height $(h)$ at $0.10 \mathrm{~mol} / \mathrm{L}$ concentration $(c)$ is 299 while the standard deviation of the noise $(\delta)$ is lower than 3 . Therefore, we estimate the detection limit (DL) of toluene to be $0.002 \mathrm{~mol} / \mathrm{L}$ with this ES-SPI method.

The mixture solution $(0.001 \mathrm{~mol} / \mathrm{L}$ each $)$ has been analyzed by a commercial ESI mass spectrometer (Thermo Finnigan LCQ Advantage MAX, Thermo Fisher Scientific, Waltham, MA, USA). No ion signal of benzene, cyclohexane, toluene, or nitrobenzene could be found with this conventional ESI method probably due to the low polarity of these molecules. In addition, no ion signal was found when this solution was analyzed with the present ES sampling method and mass spectrometer, but without the VUV light. This confirms that the signals in Figure 2 did come from the tunable synchrotron VUV photoionization of neutral molecules in the electrospray process.

The intensity of each peak correlates to the photoionization cross section and concentration of each species. In our spectrum, the intensities of peaks of aromatics are similar, and are much larger than that of cyclohexane. This is in accordance with the data of cross sections (benzene, $32 \mathrm{Mb}$; cyclohexane, $21 \mathrm{Mb}$ [14]; toluene's is close to benzene's [15]). The photoionization cross section data of nitrobenzene has not been reported. But from this spectrum, we can conjecture it is similar to that of benzene.

\section{Solid Compounds}

To demonstrate that this method can be used to analyze solid samples, here we show the results of triphenylamine, urea, and thioacetamide. Triphenylamine $(m / z=245)$ was dissolved in methanol/acetonitrile (1:4 in volume) at a concentration of $0.08 \mathrm{~mol} / \mathrm{L}$, the flow rate of solution was $40 \mu \mathrm{L} / \mathrm{min}$, the voltage applied on the ES needle was $3000 \mathrm{~V}$, the distance between the needle and the counterelectrode was $3 \mathrm{~mm}$, and the VUV photon energy was $10.33 \mathrm{eV}$. The experimental parameters for urea $(\mathrm{m} / \mathrm{z}=60)$ at $0.10 \mathrm{~mol} / \mathrm{L}$ in methanol were $20 \mu \mathrm{L} / \mathrm{min}, 2500 \mathrm{~V}, 1.5 \mathrm{~mm}$, and $10.16 \mathrm{eV}$; as for thioacetamide $(\mathrm{m} / \mathrm{z}=75)$ in methanol at $0.15 \mathrm{~mol} / \mathrm{L}$, $8 \mu \mathrm{l} / \mathrm{min}, 2450 \mathrm{~V}, 1.5 \mathrm{~mm}$, and $8.73 \mathrm{eV}$. Our experimental results show that the dominant mass peaks in the mass spectrum are from the parent ions of triphenylamine, urea, and thioacetamide. No apparent fragments were generated with this method. It is known that urea and thioacetamide can decompose easily at high temperature. Therefore, it is difficult to do a quantitative analysis of urea and thioacetamide with conventional methods that involve high temperature. The pyrolysis fragments can be avoided with this technique since no high temperature is needed. The advantage of this method over the conventional electron-impact ionization (EI) method is that the EI method produces a large amount of fragments whereas this method is almost fragment-free.
We could also measure the ionization energy (IE) of urea and thioacetamide by scanning the photon energy. The selected mass peak was integrated at different photon energy to yield the PIE spectrum, a plot of ion intensity versus photon energy. The value of IE can be obtained from the PIE spectrum directly. Here, we measured the IE values of urea and thioacetamide to be $9.81 \pm 0.02$ and $8.3 \pm 0.1 \mathrm{eV}$, respectively. These values are in good agreement with the adiabatic ionization energies via photoelectron spectroscopy [16, 17].

\section{Analysis of Petroleum Products}

This method can be applied to analyze petroleum products. The mass spectrum of a gasoline sample (7 $\mu \mathrm{L} / \mathrm{min}, 2800 \mathrm{~V}, 1.5 \mathrm{~mm}$ ) at $10.69 \mathrm{eV}$ photon energy is shown in Figure 3a. A series of peaks from $m / z=56$ to 170 are observed, which correspond to the hydrocarbons with the carbon number from $\mathrm{C}_{4}$ to $\mathrm{C}_{12}$. The mass spectrum is very similar to that of the same gasoline sample using thermal vaporization method [18], as shown in Figure $3 \mathrm{~b}$. By careful comparison, some differences can be found. The peak at $m / z=42$ is observed only in the thermal vaporization method. The peaks at $m / z=56,70,78$, and 84 are comparatively higher in the thermal vaporization method. Probably, those peaks were due to not only the components of

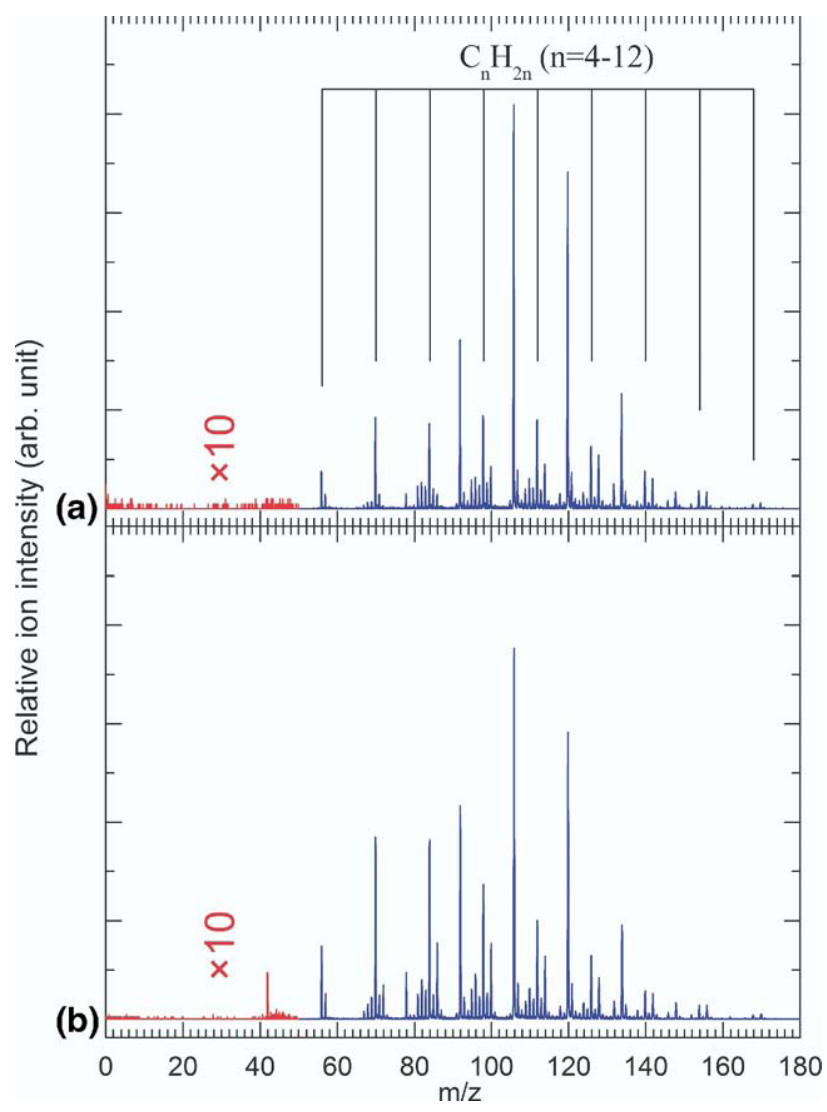

Figure 3. (a) ES-SPI mass spectrum of gasoline. (b) Thermal vaporization/VUV photoionization mass spectrum of gasoline. Both spectra were measured at $10.69 \mathrm{eV}$ photon energy. 


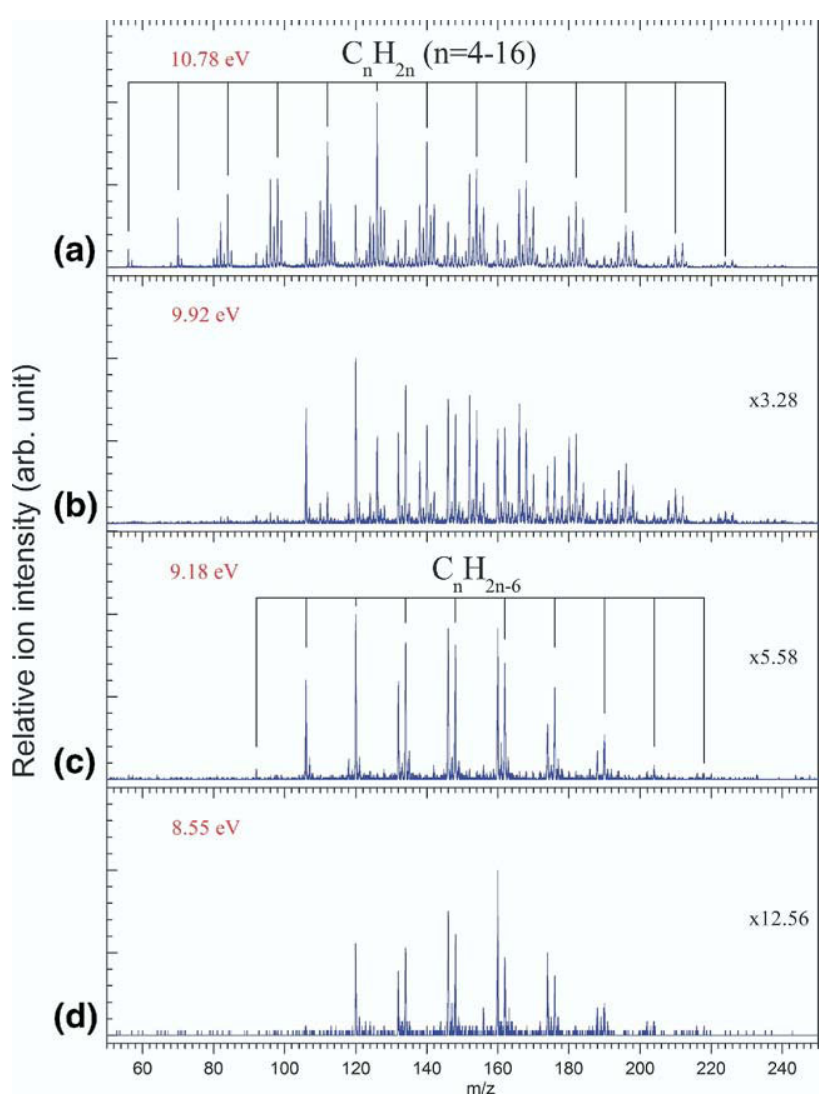

Figure 4. Normalized ES-SPI mass spectra of kerosene at (a) 10.78 , (b) 9.92, (c) 9.18, and (d) $8.55 \mathrm{eV}$ photon energies (flow rate $8 \mu \mathrm{L} / \mathrm{min}$, voltage on ES needle $2677 \mathrm{~V}$ ).

gasoline but also the thermal decomposition products from large hydrocarbon molecules.

It is difficult to analyze the components in kerosene all at once using previous methods reported [19]. It seems this ES-SPI method is slightly better at this. Figure 4 shows the ES-SPI mass spectra of the kerosene sample at (a) 10.78, (b) 9.92, (c) 9.18, and (d) $8.55 \mathrm{eV}$ photon energies $(8 \mu \mathrm{L} / \mathrm{min}, 2677 \mathrm{~V}, 1.5 \mathrm{~mm})$. As shown in Figure $4 a$, a series of peaks from $m / z=56$ to 226 are observed, corresponding to the hydrocarbons from $\mathrm{C}_{4}$ to $\mathrm{C}_{16}$, including paraffins, olefins, and aromatics. All peaks decrease gradually as the photon energy decreases. And some peaks disappear when the photon energy becomes lower than the appearance potentials (AP) of the corresponding fragment ions, or the IE values of the corresponding molecules. In Figure $4 \mathrm{c}$ and $\mathrm{d}$, there are merely aromatics peaks because of their relatively low IE values. Therefore, the major components of kerosene can be analyzed based on the mass signals at different photon energies.

By comparing the experimental PIE spectra with known PIE spectra (or known IE values) of pure substances, the individual components of kerosene can be identified. The PIE spectrum of the $\mathrm{m} / \mathrm{z}=92$ ion from the kerosene sample is shown in Figure 5a. The onset of that PIE spectrum at $8.82 \pm 0.02 \mathrm{eV}$ is close to the IE of toluene $(\mathrm{IE}=8.828 \mathrm{eV})[20]$, indicating the existence of toluene in the kerosene sample. For $m / z=106$, there might be four aromatic isomers: ethylbenzene, $o$-dimethylbenzene, $m$ dimethylbenzene, and $p$-dimethylbenzene. We could distinguish them by measuring the PIE spectrum. The PIE spectrum of $m / z=106$ from the kerosene sample is shown in Figure 5b. The onset at $8.53 \pm 0.02 \mathrm{eV}$ is close to the IE of o-dimethylbenzene $(8.56 \mathrm{eV})$ or $m$-dimethylbenzene $(8.55 \mathrm{eV})$, but different from the IE of ethylbenzene $(8.77 \mathrm{eV})$ or $p$-dimethylbenzene $(8.44 \mathrm{eV})[20]$. Thus, the kerosene sample contains $o$-dimethylbenzene and/or $m$ dimethylbenzene, probably some ethylbenzene as well. The existence of $p$-dimethylbenzene can be ruled out.

\section{Semiquantitative Analysis of Components in Gasoline}

The ion signal intensity $I_{i}$ of species $i$ in the mass spectrum can be expressed by the following equation:

$$
I_{i}=C \rho_{i} \sigma_{i}(E)
$$

where $\rho_{i}$ is the concentration of species $i, \sigma_{\mathrm{i}}(E)$ is the photoionization cross section of the species $i$ at the photon energy $E$, and $C$ is a constant for the instrument. The relative concentration $c_{i}$ of species $i$ can be expressed as:

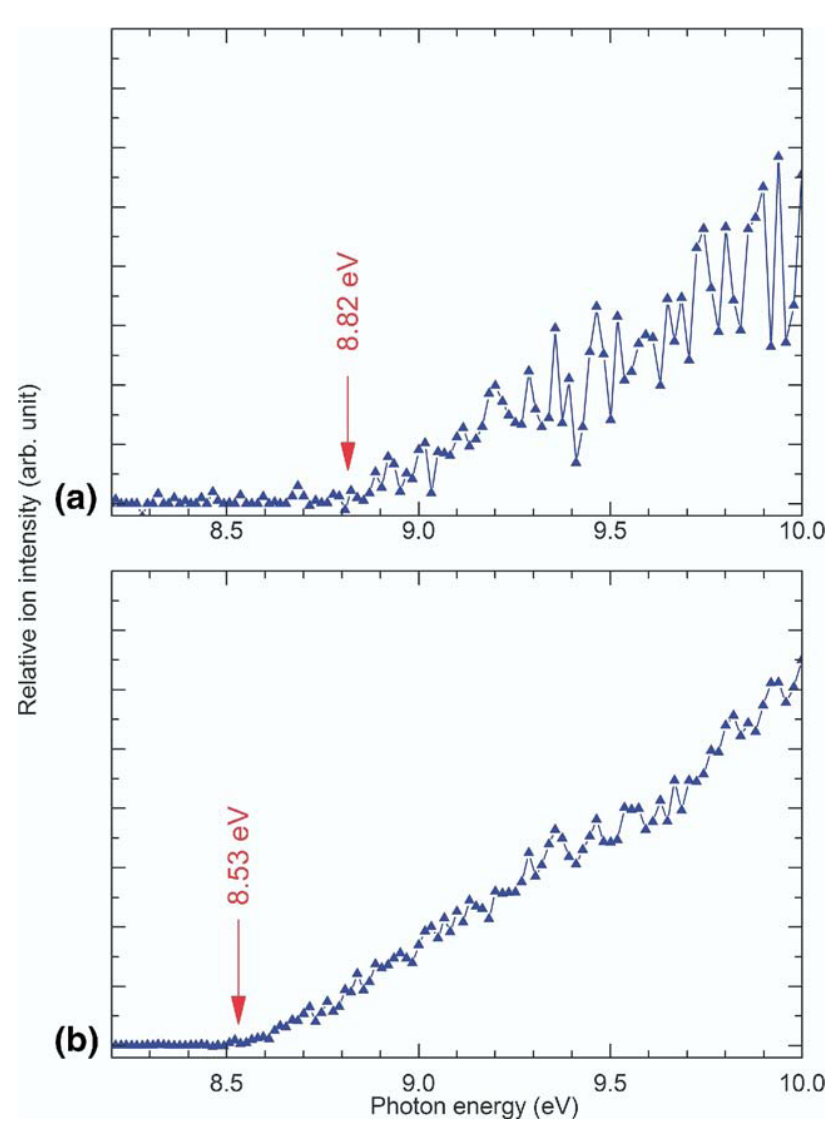

Figure 5. (a) PIE spectrum of $m / z=92$ from kerosene. (b) PIE spectrum of $\mathrm{m} / \mathrm{z}=106$ from kerosene. 


$$
c_{i}(\%)=\frac{\frac{I_{i}}{\sigma_{i}(E)}}{\sum_{i} \frac{I_{i}}{\sigma_{i}(E)}} \times 100
$$

According to our experiments, most of the components in gasoline can be ionized at $10.78 \mathrm{eV}$ photon energy. The average photoionization cross sections $\sigma_{\mathrm{i}}(E)$ at $10.78 \mathrm{eV}$ were estimated to be 4,22 , and $35 \mathrm{Mb}$ for paraffins, olefins [21], and aromatics [14], respectively. With the above equations, the relative concentrations of the even mass peaks of gasoline sample were calculated, which have been determined to be the components of gasoline by IE measurements from PIE spectra (see the Supplemental information, which can be found in the electronic version of this article). The samplespecific approach is the same as that used in reference [18]. The relative concentrations of paraffins, olefins, and aromatics were calculated to be $45.9 \%, 26.1 \%$, and $27.9 \%$, respectively. These values are close to that obtained with thermal vaporization method [18]. The discrepancy mainly comes from the uncertainties of the photoionization cross sections. These results show that our method can give an acceptable semiquantitative analysis of organic molecules in complex mixtures.

\section{Conclusions}

The method of electrospray coupled with VUV singlephoton ionization (ES-SPI) is very useful in the analysis of complex organic system without tedious pretreatment, especially for thermal labile and nonpolar compounds. Differential quantification (comparing samples) can be made without the need for knowledge of cross sections. The PIE spectra demonstrate that this technique can be used to obtain some fundamental electronic properties of thermal unstable organic species. Quantitative analysis of the components in mixtures can be achieved based on the data of IE and photoionization cross sections. The ES-SPI method might be a potential tool for the study of small biomolecules and natural products. Currently, the sensitivity of ES-SPI is relatively low compared with ESI $\left(10^{-3}\right.$ $\mathrm{mol} / \mathrm{L}$ for solid samples, but much better for volatile samples). However, there is great potential to improve it; first, better transfer efficiency can be achieved by optimizing the geometry of sample inlet interface; second, the photon flux can be enhanced by several orders of magnitude [8-10,14]. The sensitivity can be improved proportionally by optimizing these two aspects. Miniaturization of the apparatus can be achieved with the development of the VUV lamps and broadly tunable VUV laser sources. This might vastly expand the application of this method in the future.

\section{Acknowledgments}

The authors thank Miss Jing Wang, Mr. Kuiwen Zhang, Professor Zhen Gao, Professor Qihe Zhu, and Professor Weijun Zheng for many valuable discussions. The authors acknowledge support for this work by the Chinese Academy of Sciences (CAS), the National Natural Science Foundation of China under grants 20433080, 20773126, and 10705026, and the Ministry of Science and Technology of China (2007CB815204).

ZY and TCZ contributed equally to this work.

\section{References}

1. Eide, I.; Zahlsen, K. A Novel Method for Chemical Fingerprinting of Oil and Petroleum Products Based on Electrospray Mass Spectrometry and Chemometrics. Energy Fuels 2005, 19(3), 964-967.

2. Zhan, D. L.; Fenn, J. B. Electrospray Mass Spectrometry of Fossil Fuels. Int. J. Mass Spectrom. 2000, 194(2/3), 197-208.

3. Rostad, C. E. Screening of Polar Components of Petroleum Products by Electrospray Ionization Mass Spectrometry. Energy Fuels 2005, 19(3), 992-997.

4. Porter, D. J.; Mayer, P. M.; Fingas, M. Analysis of Petroleum Resins Using Electrospray Ionization Tandem Mass Spectrometry. Energy Fuels 2004, 18(4), 987-994.

5. Mysak, E. R.; Wilson, K. R.; Jimenez-Cruz, M.; Ahmed, M.; Baer, T. Synchrotron Radiation Based Aerosol Time-of-Flight Mass Spectrometry for Organic Constituents. Anal. Chem. 2005, 77(18), 5953-5960.

6. Wilson, K. R.; Belau, L.; Nicolas, C.; Jimenez-Cruz, M.; Leone, S. R.; Ahmed, M. Direct Determination of the Ionization Energy of Histidine with VUV Synchrotron Radiation. Int. J. Mass Spectrom. 2006, 249, 155-161.

7. Schramm, E.; Muehlberger, F.; Mitschke, S.; Reichardt, G.; SchulteLadbeck, R.; Puetz, M.; Zimmermann, R. Determination of the Ionization Potentials of Security-Relevant Substances with Single Photon Ionization Mass Spectrometry Using Synchrotron Radiation. Appl. Spectrosc. 2008, 62(2), 238-247.

8. Borghese, A.; Di Palma, T. M. Laser-Plasma-Based Vacuum-Ultraviolet Light Source for Tunable Single-Photon Ionization. Appl. Optics 2007, $46(22), 4948-4953$.

9. Muhlberger, F.; Wieser, J.; Ulrich, A.; Zimmermann, R. Single Photon Ionization (SPI) Via Incoherent VUV-Excimer Light: Robust and Compact Time-of-Flight Mass Spectrometer for On-Line, Real-Time Process Gas Analysis. Anal. Chem. 2002, 74(15), 3790-3801.

10. Zimmermann, R.; Welthagen, W.; Groger, T. Photo-Ionization Mass Spectrometry as Detection Method for Gas Chromatography-Optical Selectivity and Multidimensional Comprehensive Separations. J. Chromatogr. A 2008, 1184(1/2), 296-308.

11. Qi, F.; Yang, R.; Yang, B.; Huang, C. Q.; Wei, L. X.; Wang, J.; Sheng, L. S. Zhang, Y. W. Isomeric Identification of Polycyclic Aromatic Hydrocarbons Formed in Combustion with Tunable Vacuum Ultraviolet Photoionization. Rev. Sci. Instrum. 2006, 77(8), 084101.

12. GB17930-1999. In National Standard of the People's Republic of China. Standards Press of China: Beijing, 2004.

13. Muhlberger, F.; Zimmermann, R.; Kettrup, A. A mobile mass spectrometer for comprehensive on-line analysis of trace and bulk components of complex gas mixtures: Parallel application of the laser-based ionization methods VUV single-photon ionization, resonant multiphoton ionization, and laser-induced electron impact ionization. Anal. Chem. 2001, 73(15), 3590-3604.

14. Cool, T. A.; Wang, J.; Nakajima, K.; Taatjes, C. A.; McLlroy, A. Photoionization Cross Sections for Reaction Intermediates in Hydrocarbon Combustion. Int. J. Mass Spectrom. 2005, 247(1/3), 18-27.

15. Adam, T.; Zimmermann, R. Determination of Single Photon Ionization Cross Sections for Quantitative Analysis of Complex Organic Mixtures. Anal. Bioanal. Chem. 2007, 389(6), 1941-1951.

16. Bieri, G.; Asbrink, L.; von Niessen, W. 30.4-nm He (II) Photoelectron Spectra of Organic Molecules: Part VII. Miscellaneous Compounds. J. Electron Spectrosc Relat. Phenom. 1982, 27(2), 129-178.

17. Mines, G. W.; Thompson, H. W. The Photoelectron Spectra of Amides, Thioamides, Ureas, and Thioureas. Spectrochim. Acta. 1975, 31(2), 137142.

18. Wang, J.; Yang, B.; Li, Y. Y.; Tian, Z. Y.; Zhang, T. C.; QI, F.; Nakajima, K. The Tunable VUV Single-Photon Ionization Mass Spectrometry for the Analysis of Individual Components in Gasoline. Int. J. Mass Spectrom. 2007, 263(1), 30-37.

19. Blomberg, J.; Schoenmakers, P. J.; Brinkman, U. A. T. Gas Chromatographic Methods for Oil Analysis. J. Chromatogr. A 2002, 972(2), 137-173.

20. Linstrom, P. J., Mallard, W. G. NIST Chemistry WebBook; NIST Standard Reference Database Number 69, June 2005, National Institute of Standards and Technology, Gaithersburg MD; http://webbook.nist.gov.

21. Koizumi, H. Predominant Decay Channel for Superexcited OrganicMolecules. J. Chem. Phys. 1991, 95(8), 5846-5852. 